\title{
Ockhamism without Thin Red Lines
}

\author{
(postprint version) \\ Synthese 191, 2014, pp. 2633-2652
}

Andrea Iacona

This paper investigates the logic of Ockhamism, a view according to which future contingents are either true or false. Several attempts have been made to give rigorous shape to this view by defining a suitable formal semantics, but arguably none of them is fully satisfactory. The paper draws attention to some problems that beset such attempts, and suggests that these problems are different symptoms of the same initial confusion, in that they stem from the unjustified assumption that the actual course of events must be represented in the semantics as a distinguished history, the Thin Red Line.

\section{Preamble}

The term 'Ockhamism' will be used in this paper to indicate a view according to which future contingents are either true or false. To illustrate, suppose that the following sentence is uttered now:

\section{(1) It will rain}

On this view, (1) is either true or false. Whether it is true or false depends on whether it will actually rain or not. That is, (1) is true if it will actually rain, false otherwise. So it is either true or false because either it will actually rain or it will not. In this respect, there is no difference between (1) and the following sentences:

(2) It is raining

(3) It rained

(2) is either true or false as uttered now, because either it is actually raining or it is not. Similarly, (3) is either true or false as uttered now, because either it did actually rain or it did not. The truth-value of (1), just like 
the truth-value of (2) and (3), depends on what happens in one particular course of events of the many that are possible, namely, the actual course of events.

More generally, the view hinges on the idea that a future contingent 'It will be the case that $p^{\prime}$, as uttered at a time $t$, has a truth-value which depends on what happens after $t$ in the actual course of events. That is,

(O) 'It will be the case that $p$ ' is true at $t$ if and only if is true at $t$ in the actual course of events.

The view is called 'Ockhamism' because this idea goes back to Ockham's doctrine of divine foreknowledge. According to Ockham, the truth-value of every future contingent is known to God, in that it depends on what happens in the "true" future, which is one among the many possible futures. The "true" future is nothing but the actual future, that is, the future part of the actual course of events ${ }^{1}$.

A crucial feature of Ockhamism so understood is that it leaves room for a distinction between truth and determinate truth, at least if the latter is defined in the usual way as truth in all possible courses of events. (O) entails that 'It will be the case that $p$ ' is true at $t$ if it is true at $t$ in the actual course of events. But the actual course of events is just one among the many courses of events that are possible at $t$, so the truth of 'It will be the case that $p$ ' at $t$ is consistent with its falsity at $t$ in some of them. In other words, truth is a matter of actuality, while determinate truth is a matter of necessity. Determinate truth entails truth, but not the other way round. For example, (1) as uttered now may be true without being determinately true, if it will rain in the actual course of events but not in some other possible course of events ${ }^{2}$.

The thesis that future contingents are either true or false is controversial. According to a very influential line of reasoning that goes back to Aristotle, this thesis must be rejected. For the supposition that future contingents are true or false leads to the unacceptable conclusion that the future is necessary. That is, if (1) is true now then it is necessary that it will rain, and if it is false now then it is necessary that it will not rain. The distinction between truth and determinate truth provides a coherent way to resist this line of

\footnotetext{
${ }^{1} \varnothing$ hrstrøm presents Ockham's doctrine and its historical context in [15], pp. 6-10. Note that, although the view considered is reminiscent of Ockham's doctrine, this does not make it the only view that deserves to be called Ockhamism. The term 'Ockhamism' may be used in different ways, and this paper is not intended to question the legitimacy of any of them. For example, Ockhamism may be defined without reference to actuality, as the thesis that future contingents have truth-values, or that there are true future contingents. Here it is assumed, like in Øhrstrøm [14] and in Malpass and Wawer [11], that something like $(\mathrm{O})$ is essential to any view faithful to Ockham's thought.

${ }^{2}$ Here 'necessity' is understood in the historical sense, that is, as a synonym of 'settledness'. Accordingly, 'determinate truth' might also be called 'settled truth'.
} 
reasoning. If truth does not amount to determinate truth, there is no reason to assume that if (1) is true now then it is necessary that it will rain, or that if it is false now then it is necessary that it will not rain ${ }^{3}$.

Obviously, this leaves open the question of whether Ockhamism is tenable from a metaphysical point of view, that is, whether it can really stand as an account of future contingency that complies with indeterminism. Some philosophers are inclined to think that the very notion of the actual future is at odds with the indeterministic claim that many futures are equally possible. However, that question will not be addressed here. The main concern of this paper is the issue of how an adequate formal semantics can be defined if $(\mathrm{O})$ is accepted. So the paper takes for granted that it is at least consistent to think that future contingents are true or false in virtue of what happens in the actual future ${ }^{4}$.

\section{TRL Semantics}

The issue of how an adequate formal semantics can be defined if $(\mathrm{O})$ is accepted has been addressed by friends and enemies of Ockhamism. The main options that have been considered so far rest on three shared assumptions. The first - call it A1 - is that branching time structures may profitably be employed. Branching time structures are structures of the form $\langle M,<\rangle$, where $M$ is a set of moments and $<$ is a tree-like partial ordering of these moments. In such a structure, possible courses of events are represented as histories, defined as maximal linearly ordered subsets of $M$. As figure 1 shows, three moments $m_{0}, m_{1}$ and $m_{2}$ in $M$ may be such that $m_{0}<m_{1}$ and $m_{0}<m_{2}$ but it is not the case that $m_{1}<m_{2}$ or $m_{1}=m_{2}$ or $m_{2}<m_{1}$. Thus, $h_{1}$ and $h_{2}$ may be different histories such that $h_{1}$ goes through $m_{0}$ and $m_{1}$ while $h_{2}$ goes through $m_{0}$ and $m_{2}{ }^{5}$.

The second assumption - call it A2 - is that tenses are to be represented in terms of operators in the way suggested by Prior. If $p$ stands for (2), then (1) is represented as $F p$, where $F$ stands for 'It will be the case that', and (3) is represented as $P p$, where $P$ stands for 'It was the case that'. In a branching time structure, truth at a moment-history pair can be defined in this way. Let it be granted that each moment-history pair $m / h$ is formed by a moment $m$ and a history $h$ such that $m \in h$. Assuming that each propositional variable is evaluated as true or false at each moment, the definition goes as follows.

\footnotetext{
${ }^{3}$ The origin of the line of reasoning considered is Aristotle's discussion of future contingents in De interpretatione 9. Iacona [4] provides a more thorough explanation of the divergence between Ockhamism and the Aristotelian tradition, pp. 31-34.

${ }^{4}$ Iacona [4] dispels some misunderstandings that may lead to think that Ockhamism is incompatible with indeterminism in some important sense, pp. 39-44. Further objections to Ockhamism are discussed in Rosenkranz [17].

${ }^{5}$ The notion of branching time structure goes back to Kripke, see Prior [16], pp. 27-29.
} 


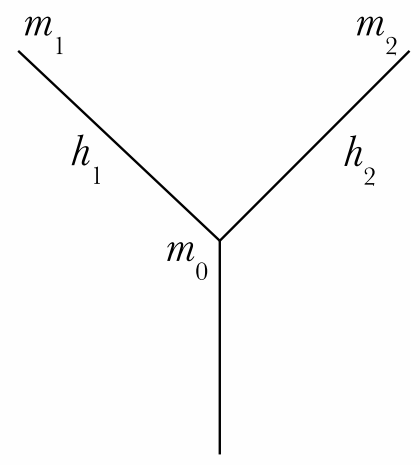

Figure 1: Branching

\section{Definition 1}

1 If $\alpha$ is an atomic formula, $\alpha$ is true at $m / h$ iff it is true at $m$;

$\mathbf{2} \sim \alpha$ is true at $m / h$ iff $\alpha$ is not true at $m / h$;

$3 \alpha \supset \beta$ is true at $m / h$ iff either $\alpha$ is not true at $m / h$ or $\beta$ is true at $m / h$;

$4 \alpha \vee \beta$ is true at $m / h$ iff either $\alpha$ is true at $m / h$ or $\beta$ is true at $m / h$;

$5 \alpha \wedge \beta$ is true at $m / h$ iff $\alpha$ is true at $m / h$ and $\beta$ is true at $m / h$;

$6 F \alpha$ is true at $m / h$ iff for some $m^{\prime}$ such that $m<m^{\prime}, \alpha$ is true at $m^{\prime} / h$;

$7 \mathrm{P \alpha}$ is true at $m / h$ iff for some $m^{\prime}$ such that $m^{\prime}<m, \alpha$ is true at $m^{\prime} / h$;

$8 \square \alpha$ is true at $m / h$ iff $\alpha$ is true at $m / h^{\prime}$ for every $h^{\prime}$ such that $m \in h$.

Falsity is defined as absence of truth: $\alpha$ is false at $m / h$ if and only if $\alpha$ is not true at $m / h^{6}$.

Let a branching time model be a model $\langle M,<, V\rangle$ formed by a branching time structure $\langle M,<\rangle$ and a valuation function $V$ constructed in accordance with definition 1. Let 'Priorian semantics' indicate the semantics characterized by branching time models, namely, the semantics that results from the combination of $\mathrm{A} 1$ and $\mathrm{A} 2$ if truth at a moment-history pair is defined in the way considered. The third assumption - call it A3 - is that actuality

\footnotetext{
${ }^{6} \mathrm{~A}$ definition of truth at a moment-history pair along these lines is provided in Prior [16], pp. 126-127.
} 
must be formally represented, that is, something must be added to Priorian semantics to make sense of $(\mathrm{O})$. A branching time model does not tell us which history is the actual history. For example, in figure 1 we do not "see" which of the two alternative future moments corresponds the state of affairs that will in fact obtain. But Ockhamism, one might think, requires that reference be made to a distinguished history as the actual history. The history in question is what Belnap and Green have called the Thin Red Line ${ }^{7}$.

The present discussion focuses on A3. Therefore, A1 and A2 will not be questioned. Since A3 implies a modification of Priorian semantics, the term 'TRL semantics' will be used to indicate the result of such modification. Thus, according to the terminology adopted here, Ockhamism and TRL semantics are not the same thing. The first is a view informally characterized, the second is a kind of formal apparatus. Perhaps this terminology does not entirely adhere to the current use of the expression 'Thin Red Line'. Sometimes, the latter is used informally as a synonym of 'actual future'. But it is important to recognize the distinction between the view under consideration and a certain kind of formal semantics. For the question to be addressed is precisely whether a semantics of that kind must be adopted to make sense of the view.

\section{Simple TRL}

TRL semantics is a kind of formal apparatus, in that there is more than one way to modify Priorian semantics in accordance with A3. This section and the next three outline the main options that have been considered so far, and draw attention to some problems that may be raised in connection with them. Each option amounts to a way of defining truth at a moment in terms of truth at a moment-history pair plus some actuality function.

The first option may be called the simple TRL, in that it assumes that truth at a moment is defined simply in terms of a unique distinguished history that is fixed once and for all in the model. Let $\langle M,<, V, A\rangle$ be a model formed by a branching time model $\langle M,<, V\rangle$ and a constant function $A$ from $M$ to the set of all histories. Call $\mathbf{h}$ the unique value of $A$. In such a model, truth at a moment may be defined as follows:

Definition $\mathbf{2} \alpha$ is true at $m$ iff $\alpha$ is true at $m / \mathbf{h}$.

Consider figure 2, where $h_{1}$ includes $m_{0}$ and $m_{1}, h_{2}$ includes $m_{0}$ and $m_{2}$, and $\mathbf{h}=h_{2}$. Suppose that $F p$ is true at $m_{0} / h_{2}$, because $p$ is true at $m_{2} / h_{2}$. Then $F p$ is true at $m_{0}$, no matter whether it is true or false at $m_{0} / h_{1}{ }^{8}$.

\footnotetext{
${ }^{7}$ This assumption is stated explicitly in Øhrstrøm [14], pp. 25-26, and in Malpass and Wawer [11], p. 124. The expression 'Thin Red Line' is introduced by Belnap and Green in [1], pp. 379-381.

${ }^{8}$ This option is considered in Belnap and Green [1], p. 379, in Belnap, Perloff and Xu [13], p. 162-163, and in MacFarlane [10], p. 325.
} 


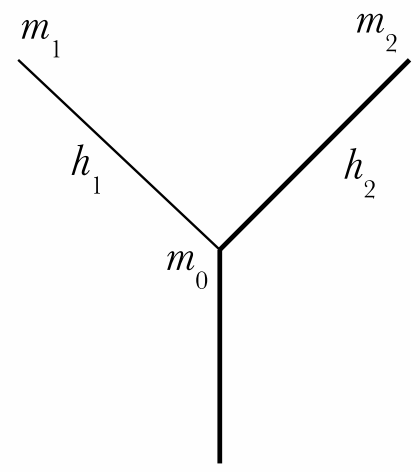

Figure 2: Branching with Thin Red Line

The main flaw of the simple TRL concerns the evaluation of formulas at moments that do not belong to $\mathbf{h}$. According to definition 2, a necessary condition for the truth of $\alpha$ at $m$ is that $m \in \mathbf{h}$. For otherwise there is no such thing as the moment-history pair $m / \mathbf{h}$. On the assumption that $\alpha$ is false at $m$ if and only if it is not true at $m$, we get that $\alpha$ is false at any moment that does not belong to $\mathbf{h}$. But this seems unacceptable, in that it blatantly violates the intended meaning of the connectives. For example, $F p$ and $\sim F p$ are both false at non-actual moments.

Note that no significant improvement is made by refining the definition of falsity. It may be assumed that $\alpha$ is false at $m$ if and only if $\alpha$ is false at $m / \mathbf{h}$, and that $\alpha$ is "undefined" if $m$ does not belong to $\mathbf{h}$. But we still get that $F p \vee \sim F p$ and $F p \wedge \sim F p$ are both undefined at non-actual moments, hence that no significant distinction can be drawn between them. Moreover, even if definition 2 is restricted to formulas of the form $F \alpha$ in order to handle cases such as those considered, some troubles remain. For example, we still get that $F(p \vee \sim p)$ is not true at non-actual moments.

Independently of the issue of the connectives, the supposition that the truth of $\alpha$ at $m$ requires that $m \in \mathbf{h}$ seems implausible. One problem that Belnap and Green have raised in connection with the simple TRL concerns the apparent truth of some counterfactual predictions. Consider figure $3 . h_{1}$ and $h_{2}$ are as in figure 2 , and $h_{3}$ is a history that includes $m_{3}$ and $m_{4}$. Let $p$ stand for (2), and suppose that it rains only at $m_{1}$. $F p$ turns out untrue at $m_{3}$, because $m_{3}$ does not belong to $\mathbf{h}$. But this seems wrong. At $m_{2}$ one might say what follows, referring to a moment like $m_{1}$ : 


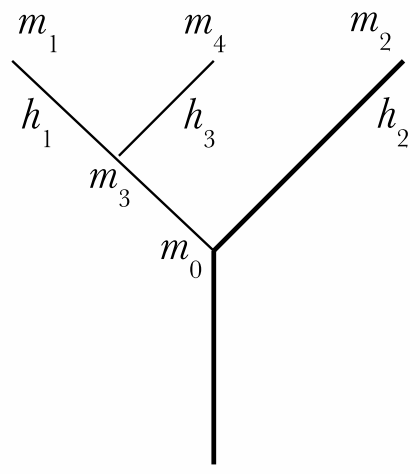

Figure 3: Counterfactual predictions

(4) Had things gone that way, one would have made a true prediction by uttering 'It will rain' before then.

There are cases in which it is plausible to regard a counterfactual prediction as true. In those cases, the intuitive evaluation of the prediction does not depend on what happens in the actual history, but rather on what happens in some other history that could be actual. In the example considered, the alternative that seems relevant to the truth of $F p$ at $m_{3}$ is whether things go as in $h_{1}$ or as in $h_{3}$. This is why $F p$ seems true on the hypothesis that $h_{1}$ is actual ${ }^{9}$.

\section{Supervaluational TRL}

The second option, which may be called the supervaluational TRL, has been suggested by Malpass and Wawer as an improvement on the first option. Malpass and Wawer provide a hybrid definition of truth at a moment which combines the notion of truth that underlies the simple TRL with the notion of truth that is characteristic of supervaluationism. According to the latter, truth at $m$ is understood as determinate truth, that is, as truth at $m / h$ for all $h$ such that $m \in h^{10}$.

Let $\langle M,<, V, A\rangle$ and $\mathbf{h}$ be as in the first option. The definition of truth at a moment now goes as follows:

\footnotetext{
${ }^{9}$ Belnap and Green [1], p. 379. See also Belnap, Perloff and Xu [13], pp. 162-163.

${ }^{10}$ The supervaluational TRL is presented in Malpass and Wawer [11]. Standard supervaluationism is outlined in Thomason [20].
} 
Definition $3 \alpha$ is true at $m$ iff either $\alpha$ is true at $m / h$ for all $h$ such that $m \in h$ or $\alpha$ is true at $m / \mathbf{h}$.

Falsity at a moment is defined in similar way. So it turns out that, if $m$ is actual and $F p$ is contingent at $m$, where 'contingent at $m$ ' means 'true at $m / h$ for some but not all $h$ such that $m \in h$ ', $F p$ is either true or false at $m$. For either it is true at $m / \mathbf{h}$ or it is false at $m / \mathbf{h}$. Instead, if $m$ is non-actual and $F p$ is contingent at $m, F p$ is neither true nor false at $m$. For there is no such thing as the moment-history pair $m / \mathbf{h}$, and $F p$ is neither true at $m / h$ for all $h$ nor false at $m / h$ for all $h$. The only case in which $m$ is non-actual and $F p$ is true (or false) at $m$ is that in which $F p$ is true (or false) in all $h$ such that $m \in h$, hence it is not contingent at $m$. According to Malpass and Wawer, this difference between actual and non-actual moments is exactly what we should expect. It is right to say that only actual predictions can be true or false, because there is no such thing as the actual future of a nonactual moment. But it is wrong to say that no sentence is true at non-actual moments. They draw a distinction between "the strong intuition", the claim that either $F p$ is true or $F \sim p$ is true at any non-actual moment, and the "the weak intuition", the claim that $F(p \vee \sim p)$ is true at any non-actual moment. While the first claim cannot be defended, the second should be maintained. It is easy to see that, for any non-actual moment $m, F(p \vee \sim p)$ turns out true at $m / h$ for all $h$, given that, in every history, $p \vee \sim p$ is true at every moment ${ }^{11}$.

The supervaluational TRL definitely fares better than the simple TRL when it comes to the preservation of truths that seem grounded on general modal facts, such as $F(p \vee \sim p)$. Yet this does not make it an ideal option. Some doubt may be raised about the hybrid nature of definition 3, which involves a combination of truth and determinate truth, and its effects on the understanding of the connectives. Definition 3 says that a sentence is true if and only if either it is determinately true or it is true in the actual history. Therefore, we get that sometimes - at actual moments - the sentence is true because it is true in the actual history (in virtue of the second disjunct), while at other times - at non-actual moments - it is true because it is true in all histories (in virtue of the first disjunct). This ambivalence emerges clearly if we consider the following disjunction:

(5) Either it will rain or it will not rain

From definition 3 it turns out that (5) may be true at different moments for different reasons, so to say. Let us assume that $m$ is actual, that $m^{\prime}$ is non-actual, and that $F p$ and $F \sim p$ are contingent both at $m$ and at $m^{\prime}$. According to definition 3, $F p \vee F \sim p$ is true at $m$, because it is true at $m / \mathbf{h}$. Since the truth of $F p \vee F \sim p$ at $m / \mathbf{h}$ depends on either $F p$ or $F \sim p$

\footnotetext{
${ }^{11}$ See Malpass and Wawer [11], pp. 131-133.
} 
being true at $m / \mathbf{h}$, it seems correct to say that $F p \vee F \sim p$ is true at $m$ because one of its disjuncts is true at $m$. However, the same does not hold for the truth of $F p \vee F \sim p$ at $m^{\prime}$. According to definition 3, $F p \vee F \sim p$ is true at $m^{\prime}$, because it is true at $m^{\prime} / h$ for all $h$. This is due to the fact that, for any $h$ through $m^{\prime}$, either $F p$ or $F \sim p$ is true at $m^{\prime} / h$. But neither $F p$ nor $F \sim p$ is true at $m^{\prime} / h$ for all $h$, given that $F p$ and $F \sim p$ are contingent at $m^{\prime}$. Therefore, even if $F p \vee F \sim p$ is true at $m^{\prime}$, neither $F p$ nor $F \sim p$ is true at $m^{\prime}$. As it is well known, the supervaluational notion of truth involves denial of truth-functionality for disjunction: a disjunction can be true even if neither of its disjuncts is true. The result we get is that, while $F p \vee F \sim p$ is true at $m$ because one of its disjuncts is true at $m, F p \vee F \sim p$ is true at $m^{\prime}$ for a different reason, namely, that it is true at $m^{\prime} / h$ for all $h$, in spite of the fact that neither of its disjuncts is true at $m^{\prime}$. So it is natural to ask how the connective $\vee$ is to be understood. There are two ways to go, neither of which looks promising. One is to say that $\vee$ is truth-functional at actual moments and non-truth-functional at non-actual moments. But in this case one must be willing to accept that the meaning of a logical constant varies with the moment of evaluation. The other is to say that $V$ has a unique non-truth-functional meaning, although its non-truth-functionality is not manifest at actual moments. But in this case the truth of a disjunction is explained in terms of determinate truth, which is definitely not in the spirit of Ockhamism as it is understood here. What Ockhamism should entail is that (5) is true because one of its disjuncts is true, or so it will be assumed.

Further misgivings concern the problem of counterfactual predictions. To motivate their distinction between actual and non-actual predictions, Malpass and Wawer claim that there is no such thing as the actual future of a non-actual moment. They use the following example. Imagine that I hold a coin in my hand. I do not flip the coin but I could have done so. I could have said, before the possible toss, that the coin would show heads. But since the coin wasn't flipped, this assertion cannot be counted as true. The same goes for the assertion that the coin would show tails. As they say, "Each result is just an unactualised possibility for a fair coin toss that never happened" 12 .

However, if one is apt to doubt their claim, one will hardly be convinced by their example. Certainly, the coin has not been tossed, so each result is an unactualised possibility. Yet this does not entail that there is no answer to the question of what would have happened if the coin had been tossed. For what really matters to that question is not which possibility is actual, but which possibility would be actual. There is a clear sense in which one of the two results could be actual, and this seems to be the sense we have in mind when we ask that question. Therefore, the same goes for the question of whether the counterfactual prediction is true. For it is plausible that, just

\footnotetext{
${ }^{12}$ Malpass and Wawer [11], p. 129.
} 
as the truth of an actual prediction depends on what will happen, the truth of a counterfactual prediction depends on what would happen if certain conditions were to obtain.

Note also that, in the example of the coin toss, the counterfactual prediction concerns two possible outcomes whose odds are perfectly even: the probability of tails and heads is exactly the same in the counterfactual circumstances imagined. This might bolster the impression that there is no answer to the question of what would have happened if the coin had been tossed. However, if we imagine counterfactual circumstances that differ from actual circumstances precisely in that the probability for a certain possible outcome is significantly higher, or lower, that impression loses force. For example, if it is actually sunny and we imagine a counterfactual situation in which it is about to rain, it is even less plausible to say that there is no answer to the question of what would happen then.

The force of the problem of counterfactual predictions lies in the fact that we are able to conceive counterfactual circumstances and intelligibly wonder what would happen in those circumstances. Thinking about counterfactual situations involves the capacity to reason from the point of view of possible courses of events that differ from ours. Consider figure 3. Although we know that $h_{2}$ is the actual history, we can entertain the hypothesis that things have gone differently, and wonder what would happen after $m_{3}$. If $h_{1}$ were the actual history, $m_{1}$ would obtain, hence $F p$ would be true at $m_{3}$. This is not to suggest that what is at issue here is the truth-value of certain subjunctive conditionals phrased in the object language. As a matter of fact, the supervaluational TRL, just like the simple TRL, does not contemplate a counterfactual connective, so its implications on the semantics of such a connective are irrelevant for the present purposes. The point is rather that certain results concerning simple formulas of the object language seem to conflict with certain thoughts that we can express in the metalanguage by means of constructions in subjunctive form ${ }^{13}$.

\section{The Rigidity Problem}

The troubles that affect the simple TRL and the supervaluational TRL seem to stem from a common source. If the actual history is fixed once and for all in the model, it turns out that sentences lose some basic semantic properties when they are evaluated at non-actual moments. However, there is a sense in which one may expect that all moments are alike with respect to those properties, namely, the sense in which every moment is actual from its own point of view. The inability to account for this sense may be called the rigidity problem.

\footnotetext{
${ }^{13}$ Or at least, this is what Belnap and Green seem to grant in [1] when they use examples such as (4).
} 
The rigidity problem concerns the ascription of actuality to possible courses of events that differ from ours: when a non-actual moment is thought as actual, it is natural to suppose that it has its own actual future, so that it belongs to some actual course of events. Therefore, one way to bring the problem into focus is to adopt Lewis' distinction between primary and secondary sense of 'actual'. According to this distinction, in the primary sense 'actual' rigidly refers to our world, as in 'This city could be bigger than it actually is', while in the secondary sense its reference shifts from our world to a different world that is under consideration, as in 'If this city were bigger, it would actually have more buildings'. Consider figure 3 . If one is in $h_{2}$, it seems that one can meaningfully ask about a moment like $m_{3}$ :

(6) If things were so, what would actually happen?

On a natural reading of (6), 'actually' refers to some history other than $h_{2}$, that is, some history that includes $m_{3}$. But there is no way to make sense of this reading if it is assumed that the only history to which actuality can be ascribed is $h_{2}{ }^{14}$.

Of course, one might deny Lewis' distinction and claim that there is no secondary sense of 'actual' in need of account. This amounts to saying that in (6) 'actually' refers to $h_{2}$, despite appearance to the contrary. But in that case one would have to show that there is no relevant semantic difference between 'would' and 'will', hence that they can be substituted in (6) without changing its content. Moreover, one would have to explain how similar cases can be handled. For example, if 'then' is added in (6) after 'happen', it is hard to see what it could mean on the suggested reading of 'actually'. Unless one is willing to take this route, one must recognize that a semantics of the kind considered misses something.

It is important to understand that this shortcoming does not concern the mere possibility of distinguishing two senses of 'actual'. The rigidity problem does not amount to the inability to define a sense of 'actual' that is not rigid. Nothing prevents us from thinking that a TRL semantics with fixed actual history can leave room for some understanding of 'actual' that mimics the indexical account of actuality. The problem is rather that such a semantics seems incapable of making sense of the counterfactual hypothesis that one of the courses of events that include a given non-actual moment is the actual course of events, so it contains the actual future of that moment ${ }^{15}$.

This becomes clear clear if one reflects on how an actuality operator could be defined in the simple TRL or in the supervaluational TRL. As the actual history is fixed once and for all in the model, a natural way to go is

\footnotetext{
${ }^{14}$ The distinction between primary and secondary sense of 'actual' is drawn in Lewis [8], p. 19.

${ }^{15}$ The indexical account of actuality is the account suggested in Lewis [7].
} 
the following:

Definition $4 @_{1} \alpha$ is true at $m / h$ iff $\alpha$ is true at $m / \mathbf{h}$.

The operator $@_{1}$ is rigid, in that the only history that matters to the truth of $@_{1} \alpha$ at any moment-history pair is $\mathbf{h}$. Definition 4 entails that, no matter whether definition 2 or definition 3 is adopted, $@_{1} \alpha$ is true at $m$ if and only if $\alpha$ is true at $m / \mathbf{h}$, hence it is false at $m$ if $m$ is not in $\mathbf{h}$. But this is not the only way to go. An alternative definition is the following:

Definition $5 @_{2} \alpha$ is true at $m / h$ iff $\alpha$ is true at $m / h^{16}$.

Although the distinction between $@_{1}$ and $@_{2}$ makes no difference if definition 2 is adopted, it does make a difference if definition 3 is adopted. For if $m$ is not in $\mathbf{h}$ and $\alpha$ is true at $m$ in virtue of the first disjunct of definition 3 , we get that $@_{2} \alpha$ is true at $m$. Therefore, at least in the supervaluational TRL, it is clear that there are two ways to understand 'actual' one of which is not rigid. What is not clear, however, is whether $@_{2}$ captures what we want. Since the only case in which $@_{2} \alpha$ is true at $m$ for non-actual $m$ is that in which $\alpha$ is true at $m / h$ for all $h$, it turns out that $@_{2}$ expresses necessity at $m$, that is, truth in all courses of events that are possible at $m$. Yet actuality $m$ and necessity at $m$ are not the same thing. Let it be granted that the question of what will actually happen differs from the question of what will necessarily happen, as Ockhamism requires. Then it is hard to see why the question of what would actually happen in certain circumstances should not differ from the question of what would necessarily happen in those circumstances. Apparently, the expression 'what would actually happen' that occurs in (6) is not synonymous with 'what would necessarily happen'. In order to show that the supervaluational TRL can handle the rigidity problem, one should either argue against this appearance, or provide an alternative definition of the actuality operator.

\section{Relative TRL}

How can TRL semantics get over the rigidity problem? A first thought that may come to mind is that there is a simple way to account for counterfactual ascriptions of actuality if each model has a fixed actual history, that is, by considering different models. Suppose that our intended model $\mathcal{M}$ is as in figure 3 , so that $\mathbf{h}=h_{2}$. In order to represent the counterfactual hypothesis that $h_{1}$ is the actual history, it suffices to consider another model $\mathcal{M}^{\prime}$ that differs from $\mathcal{M}$ only in that $\mathbf{h}=h_{1}$, as in figure 4 . In $\mathcal{M}^{\prime}$ the counterfactual prediction expressed in (4) can be evaluated as true. More generally, for any non-actual moment in any given model, there is another model in which that moment is actual, so the same sentence can be evaluated at a moment relative to different models.

\footnotetext{
${ }^{16}$ García-Carpintero considers a definition along these lines in [3], p. 11.
} 


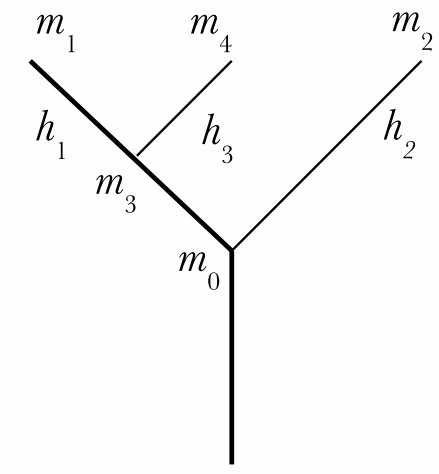

Figure 4: The relativization strategy

There is nothing inconsistent in this thought. Sentences can be evaluated at moments relative to different models, that is, models that differ only in the value of $A$. The fact, however, is that relativity to models so understood is equivalent to relativity to histories. For example, there is no substantial difference between saying that $F p$ is true at $m_{0}$ relative to $\mathcal{M}$ but false at $m_{0}$ relative to $\mathcal{M}^{\prime}$ and saying that $F p$ is true at $m_{0} / h_{2}$ but false at $m_{0} / h_{1}$. This means that what can be expressed in a TRL semantics in terms of relativity to models can equally be expressed in Priorian semantics without postulating any actual history, hence that TRL semantics boils down to an unnecessarily convoluted variant of Priorian semantics ${ }^{17}$.

A different move is to reject the idea that the actual history must be fixed once and for all in the model, and assume that the function $A$ in $\langle M,<, V, A\rangle$, instead of being constant, assigns to each moment $m$ a history $A(m)$ that satisfies the following minimal condition:

$\left(\mathrm{C}_{1}\right) m \in A(m)$

On this assumption, the definition of truth at a moment may be rephrased as follows:

Definition $6 \alpha$ is true at $m$ iff $\alpha$ is true at $m / A(m)$.

The hypothesis that at $m_{3}$ things go as in $h_{1}$ is now represented as the hypothesis that $A\left(m_{3}\right)=h_{1}$, as in figure 4 . So $F p$ turns out true at $m_{3}$ on

\footnotetext{
${ }^{17}$ Malpass and Wawer are well aware of this, see [11], p. 128.
} 
that hypothesis ${ }^{18}$.

This option, which may be called the relative $T R L$, is not affected by the rigidity problem, as it implies that every moment has its own actual future. One way to see how it differs from the simple TRL and the supervaluational TRL is to consider again the operator $@_{2}$. If definition 5 is adopted, it turns out that $@_{2} \alpha$ is true at $m$ if and only if $\alpha$ is true at $m / A(m)$. Therefore, $@_{2}$ expresses a plausible non-rigid sense of 'actual' which does not entail that actuality at $m$ and necessity at $m$ are the same thing. For example, if $A\left(m_{3}\right)=h_{1}$, as in figure 4 , then $@_{2} F p$ is true at $m_{3}$, even if $F p$ is not true at $m_{3} / h$ for all $h$.

However, as Belnap and Green have argued, the relative TRL has some undesirable consequences. Consider the following condition on $A$ :

$\left(\mathrm{C}_{2}\right)$ If $m<m^{\prime}$ then $A(m)=A\left(m^{\prime}\right)$.

On the one hand, it seems that $\left(\mathrm{C}_{2}\right)$ should not hold. For the conjunction of $\left(\mathrm{C}_{1}\right)$ and $\left(\mathrm{C}_{2}\right)$ is inconsistent with branching. Suppose that $m<m^{\prime}$ and $m<m^{\prime \prime}$. From $\left(\mathrm{C}_{2}\right)$ it follows that $A(m)=A\left(m^{\prime}\right)$ and $A(m)=A\left(m^{\prime \prime}\right)$, so that $A\left(m^{\prime}\right)=A\left(m^{\prime \prime}\right)$. Consequently, from $\left(\mathrm{C}_{1}\right)$ we get that $m^{\prime} \in A\left(m^{\prime}\right)$ and $m^{\prime \prime} \in A\left(m^{\prime}\right)$, which entails that that either $m^{\prime}<m^{\prime \prime}$ or $m^{\prime}=m^{\prime \prime}$ or $m^{\prime \prime}<m^{\prime}$. On the other hand, if $\left(\mathrm{C}_{2}\right)$ does not hold, then a principle such as the following must be rejected:

(P) If $\alpha$ is true at $m$, then $F \alpha$ is true at $m^{\prime}$ for any $m^{\prime}$ such that $m^{\prime}<m$.

Suppose that $A\left(m_{3}\right)=h_{1}$, as in figure 4 , and that $A\left(m_{0}\right)=h_{2}$, as in figure 3. Suppose also that it rains only at $m_{1}$. Then $F p$ is true at $m_{3}$, because it is true at $m_{3} / h_{1}$. But $F F p$ is false at $m_{0}$, because it is false at $m_{0} / h_{2}$. Belnap and Green claim that the failure of $(\mathrm{P})$ is not a desirable result, and here it will assumed that an Ockhamist should agree on this. If (1) is true at a given time in the actual course of events, at any previous time it must be true in the actual course of events that (1) will be true in the actual course of events ${ }^{19}$.

Against Belnap and Green, Øhrstrøm has contended that there is no reason to expect that the function $A$ satisfies $\left(\mathrm{C}_{2}\right)$. Instead of $\left(\mathrm{C}_{2}\right)$, a weaker

\footnotetext{
${ }^{18}$ Braüner, Hasle and Øhrstrøm propose a semantics of this kind in [19] and in [18]. A different version, suggested by McKim [12], is that in which the value of the function for $m$ is a linearly ordered set of moments that starts with $m$, rather than a whole history. Belnap, Perloff and Xu [13] considers both options, pp. 165-168.

${ }^{19}$ This is essentially the problem raised in Belnap and Green [1], p. 380, and in Belnap, Perloff and $\mathrm{Xu}$ [13], pp. 166-167, with some differences of formulation due to definitions 1 and 6 . The assumption that an Ockhamist should preserve $(\mathrm{P})$ depends on the understanding of Ockhamism adopted in this paper, so it does not rule out that a formal semantics inspired by the ideas of Ockham, such as that proposed by Braüner, Hasle and $\varnothing$ hrstrøm, can coherently reject (P).
} 
condition should be required, that is,

$\left(\mathrm{C}_{2}{ }^{*}\right)$ If $m<m^{\prime}$ and $m^{\prime} \in A(m)$, then $A(m)=A\left(m^{\prime}\right)$

Øhrstrøm outlines a semantics in which $A$ is defined in accordance with $\left(\mathrm{C}_{2}{ }^{*}\right)$, and a notion of "counterfactual branch" is defined in terms of $A$ as follows: for any $m$, a counterfactual branch at $m$ is a history $h$ such that $m \in h$ and $A\left(m^{\prime}\right)=h$ for any $m^{\prime}$ such that $m^{\prime} \in h$ and $m<m^{\prime}$. In this semantics, truth is defined relative to moment-history pairs by adopting the notion of counterfactual branch as a constraint on the set of moment-history pairs. That is, a formula $\alpha$ is true at $m / h$ for any counterfactual branch $h$ at $m$ in the way shown by definition $1^{20}$.

The semantics proposed by Øhrstrøm certainly has some plausible implications. For example, it preserves the validity of the schemas $P P \alpha \supset P \alpha$, $F F \alpha \supset F \alpha$ and $\alpha \supset P F \alpha$. The third schema may be regarded as a way to express the thought that underlies $(\mathrm{P})$. What is not clear, however, is whether this semantics can be regarded as a form of TRL semantics rather than a variant of Priorian semantics, given that truth is defined simply relative to moment-history pairs. The central idea of TRL semantics as understood here is that, in addition to a definition of truth at a momenthistory pair, a definition of truth at a moment must be given in terms of some actuality function ${ }^{21}$.

This suggests a more general reflection about the relativization strategy. In order to overcome the rigidity problem, one must find a way to account for counterfactual ascriptions of actuality. But there is no obvious way to make room for actuality shifts - in terms of relativity to models or in terms of relativity to histories within the same model - without ending up with a formal apparatus that bears a very close resemblance to Priorian semantics. This is what may be called the triviality problem: it is unclear whether there is a substantial difference between having many actual histories and having no actual history.

\section{$7 \quad$ Plain truth and truth in a history}

As the previous sections show, it is not clear how an adequate TRL semantics can be defined. On the one hand, if the actual history is fixed once and for all in the model, the semantics runs into the rigidity problem. On the other, the route of relativization leads to the triviality problem, at least if certain undesirable consequences are to be avoided. So the dilemma seems to be

\footnotetext{
${ }^{20}$ This proposal is defended in $\varnothing$ hrstrøm [14], pp. 29-30, and is already present in Braüner, Hasle and Øhrstrøm [18], pp. 204-205.

${ }^{21}$ Independently of this issue, Øhrstrøm's proposal has some unorthodox consequences, as explained in [14], p. 30.
} 
the following: either the formal representation of actuality is incomplete, in that it misses something important, or it does not add anything interesting to what Priorian semantics is able to express. Perhaps there is a way out of this dilemma, through some amended version of TRL semantics that has not yet been explored. But independently of whether such a version exists, it is legitimate to ask whether there are reasons to think that if no adequate TRL semantics is provided, then Ockhamism is untenable. In this section it will be suggested that there is no such reason. Contrary to what has been taken for granted, Ockhamism can be maintained without assuming that actuality must be formally represented.

The crux of the matter is the relation between plain truth, which is a property that belongs to sentences of natural language (relative to times), and truth in a history, which is defined in rigorous way for formulas of a formal language (relative to moments). Ockhamism provides an analysis of plain truth, that is, $(\mathrm{O})$. The thought that underlies $(\mathrm{O})$ is that, when a future contingent is uttered at $t$, the utterance involves reference to one in particular among the many courses of events that are possible at $t$, the actual course of events. One way to substantiate this thought is to embrace the indexical account of actuality, and say that the actual course of events is part of the context of utterance because it is the course of events in which the utterance occurs. But that is not the only way. Even if it is not assumed that actuality is indexical, it is still consistent to claim that the utterance of a future contingent involves reference to the actual course of events.

According to $(\mathrm{O})$, plain truth concerns a single course of events, the actual course of events. Therefore, a formal semantics in which truth is defined for a set of histories can provide a characterization of plain truth to the extent that one of the histories in the set represents that course of events. To illustrate, suppose that (1) is uttered now. The following instance of $(\mathrm{O})$ provides the truth-condition of the utterance:

(7) (1) is true now if and only if it is true now in the actual course of events.

The right-hand side of (7) says that (i) there is a possible course of events $c$ such that (1) is true now in $c$, and (ii) $c$ is the actual course of events. A formal semantics can provide a precise characterization of (i) to the extent that, in some model $\mathcal{M}$ in which a history $h$ represents $c$ and a moment $m$ in $h$ represents the present time, a formula that stands for (1) is true at $m / h$. Given such characterization, (ii) may be understood as a hypothesis about $\mathcal{M}$. That is, $\mathcal{M}$ gives us an account of the truth of (1) on the hypothesis that $h$ is the actual history. (ii) need not be expressed in the semantics, because it is a hypothesis about the semantics.

The plausibility of this line of thought turns out clear if one thinks about modality in its most elementary form. Consider the standard semantics of 
propositional modal logic. Anyone will agree that the notion of truth at a world adopted in that semantics does not provide an account of plain truth. The underlying assumption is that plain truth involves something more than what is represented in the semantics in terms of that notion, namely, actuality. That is,

(T) A sentence is true if and only if it is true in the actual world.

The semantics provides a precise characterization of what it is for a sentence to be true in a world, but it need not specify which world is the actual world. The fact is that truth at a given world in a model offers a representation of plain truth on the hypothesis that the world in question is the actual world. There is no reason to think that the explanatory role of the notion of truth in a history differs in this respect from the explanatory role of the notion of truth at a world. A possible course of events is nothing but a world, so (O) may be regarded as a special case of $(\mathrm{T})^{22}$.

If the relation between plain truth and truth in a history is understood in the way suggested, it turns out clear that the notion of truth in a history can be adopted as part of the Ockhamist analysis of plain truth. The remaining part of the analysis concerns a fact about natural language that need not be expressed in a formal semantics, namely, the fact that the utterance of a future contingent at a time $t$ involves reference to one among the many courses of events that are possible at $t$, the actual course of events. So there seems to be no reason to assume that actuality must be formally represented.

Note that this conclusion affects not only the varieties of TRL semantics considered in the previous sections, where an actuality function occurs in the definition of truth, but also any formal semantics in which such a function is employed to provide an interpretation of the operator $F$. If the fact that the utterance of a future contingent involves reference to the actual course of events need not be expressed formally, there is no reason to define $F$ in terms of an actuality function. Just as Ockhamism requires no modification of the standard notion of truth in a history, it requires no modification of the standard interpretation of $F$.

\section{Ockhamism and Priorian semantics}

The foregoing considerations suggest that branching time models suffice for Ockhamism. For branching time models are models whose valuation function is constructed in accordance with definition 1 , which involves the standard notion of truth in a history and the standard interpretation of $F$.

\footnotetext{
${ }^{22}$ Obviously, the semantics need not specify which world is the actual world insofar as the language does not contain an actuality operator such as $@_{1}$, that is, an actuality operator whose definition involves reference to a distinguished world.
} 
Since Priorian semantics has been defined as the semantics characterized by branching time models, this means that Priorian semantics can consistently be associated with Ockhamism ${ }^{23}$.

To get a better grasp of this possibility, consider again figure 1 as an illustration of a branching time model in which $m_{0}$ is the present time. As noted in section 2 , in figure 1 we do not "see" which of the two alternative future moments $m_{1}$ and $m_{2}$ corresponds to the state of affairs that will in fact obtain: the model does not tell us which history is the actual history. However, if one accepts $(\mathrm{O})$, one can reason under the hypothesis that one of the two histories is the actual history. Suppose for example that $F p$, which stands for (1), is true at $m_{0} / h_{2}$ but false at $m_{0} / h_{1}$. Then, if $h_{2}$ is the actual history, the truth of $F p$ at $m_{0} / h_{2}$ represents the truth of (1) as uttered now, in accordance with $(\mathrm{O})$. That is, (1) as uttered now is represented as true on the hypothesis that $h_{2}$ is the actual history. Similarly, (1) as uttered now may be represented as false on the hypothesis that $h_{1}$ is the actual history.

As long as it is granted that any consideration about actuality concerns the interpretation of a branching time model, rather than the model itself, none of the problems discussed in the previous sections can arise. Let us begin with the two problems raised in connection with the simple TRL. In the first place, Priorian semantics causes no trouble with the connectives. Even if one supposes that a given history in a branching time model is the actual history, that supposition has no consequence on the evaluation of formulas in other histories. In a branching time model, each formula is evaluated in the same way in any history. Secondly, Priorian semantics rules out the problem of counterfactual predictions. As far as a branching time model is concerned, there is no difference between thinking about actual circumstances and thinking about counterfactual circumstances. When one thinks about counterfactual circumstances, just as when one thinks about actual circumstances, one reasons under the hypothesis that one of the histories in the model is the actual history. The only difference is that one picks up a different history. Consider figure 1 . Even if it is assumed that $h_{2}$ is the actual history, so that (1) as uttered now is true, it is legitimate to say that, if $h_{1}$ were the actual history, (1) as uttered now would be false. This amounts to saying that (1) as uttered now is false on the hypothesis that $h_{1}$ is the actual history. Similar considerations hold for cases such as (4). If a history in which it rains at a certain moment is the actual history, then a counterfactual prediction made by uttering (1) before that moment is true. For $F p$ turns out true if evaluated at previous moments in the same history.

The two problems raised in connection with the supervaluational TRL are rooted out as well. In the first place, if one adopts Priorian semantics

\footnotetext{
${ }^{23}$ Interestingly, the thought that Priorian semantics suits Ockhamism lies at the origin of Priorian semantics, as it is shown by the fact that Prior himself called 'Ockhamist' that semantics in Prior [16], pp. 126-127.
} 
as suggested, one does not get the anomaly that a sentence may be true at different moments for different reasons. Consider (5). In a branching time model, (5) as uttered now is represented as true insofar as $F p \vee F \sim p$ is true at some $m / h$ and $h$ is taken to be the actual history. Since $F p \vee F \sim p$ is true at $m / h$ if and only if either $F p$ or $F \sim p$ is true at $m / h$, the only way in which (5) can be true is that in which its truth depends on the truth of one of its disjuncts. For example, in the model illustrated in figure 1, (5) as uttered now turns out true on the hypothesis that $h_{2}$ is the actual history. For $F p \vee F \sim p$ is true at $m_{0} / h_{2}$. Since $F p \vee F \sim p$ is true at $m_{0} / h_{2}$ because $F p$ is true at $m_{0} / h_{2}$, we get that (5) as uttered now is true because (1) as uttered now is true. Secondly, no controversial claim about counterfactual predictions need be made, given that the problem of counterfactual predictions is ruled out from the very beginning. Thus, in the case of the possible coin toss, there is no need to deny that there is an answer to the question of what would have happened if the coin had been tossed. More generally, if Priorian semantics is adopted, the rigidity problem does not arise. In a branching time model, just as one can make sense of the thought that one of the possible courses of events that include the present moment is the actual course of events, one can equally make sense of the counterfactual supposition that one of the possible courses of events that include a given non-actual moment is the actual course of events.

The problems raised in connection with the relative TRL do not differ in this respect from those raised in connection with the simple TRL and the supervaluational TRL. The dilemma that comes out of the discussion of the options considered in section 6 is that either the relative TRL generates undesirable consequences, or it runs into the triviality problem. But it is easy to see that, if Priorian semantics is adopted, this dilemma falls down. For it can only hold for a semantics in which a certain kind of actuality function is employed in the definition of truth.

In substance, if Ockhamism is combined with Priorian semantics, the problems discussed in the previous sections do not arise. The important point that emerges from this observation is that the problems discussed in the previous sections do not concern Ockhamism per se. They concern TRL semantics, which is a kind of formal semantics that can be associated with Ockhamism. Therefore, it would be wrong to assume, as it is often assumed in the current debate on future contingents, that these problems provide reasons against Ockhamism. If there are reasons to reject Ockhamism, they must be different reasons.

\section{Two interpretations of Priorian semantics}

Priorian semantics, as any formal apparatus, can be understood in more than one way. The Ockhamist interpretation considered in the previous section 
is one of them. But there are others. In particular, there is at least one clear alternative to the Ockhamist interpretation, namely, the interpretation according to which there is no such thing as truth simpliciter for future contingents, because there is no such thing as the actual future. Thomason draws a distinction between the two interpretations as follows:

The difference between these two is explained most clearly by supposing that we occupy the time $\alpha$ and are seeking to evaluate certain predictions made in $\alpha$. According to the first interpretation we do this by provisionally positing a possible future for $\alpha$, because a prediction made at $\alpha$ can only be true or false relative to such a future. According to the second interpretation, just one of the possible futures for $\alpha$ is the right one - the one that will be actualized ${ }^{24}$.

The "second interpretation" is the Ockhamist interpretation. The "first interpretation", instead, seems very close to a view that substantively differs from Ockhamism, the view defended by Belnap, Perloff and Xu. According to that view, which may be called "contextual pluralism", a future contingent as it is uttered in a context can be evaluated as true or false only relative to this or that possible future. For no single possible course of events is fixed by the context as the unique possible course of events that matters to the evaluation ${ }^{25}$.

Since this paper makes no attempt to defend Ockhamism, it is not among its purposes to argue against contextual pluralism. The only thing that must be clear is that the two views provide different but equally legitimate ways to understand Priorian semantics. Therefore, it would be wrong to postulate a necessary connection between Priorian semantics and one of them. To see the relevance of this qualification, consider the following remarks by Malpass and Wawer on the history parameter in Priorian semantics:

We accept that there are no technical problems with how this parameter works, but we argue that there are difficulties when trying to understand what this semantic parameter means. The value has to be given somehow and a first thought is that it is fixed by reference to some fact, or set of facts. But which facts? The obvious answer is: any set of facts that includes the fact about which future is the actual one. However, proponents of Priorian-Ockhamism strongly deny that there is any such fact; to them there is no such thing as the actual future. So, we can't

\footnotetext{
${ }^{24}$ Thomason [20], pp. 270-271. Thomason finds both interpretations objectionable, although he recognizes that his arguments do not provide a final refutation of Priorian semantics, see fn. 9.

${ }^{25}$ Belnap, Perloff and Xu [13], p. 232.
} 
simply give the history parameter the value of the actual history, as there is none (as Belnap et al. urge) ${ }^{26}$.

Here Malpass and Wawer seem to identify Priorian semantics with a view according to which there is no such thing as the actual future. But if the distinction between Priorian semantics and its interpretations is recognized, it turns out clear that what they say is questionable. If the term 'PriorianOckhamism' refers to Priorian semantics, then it is simply false that a proponent of Priorian-Ockhamism must deny that the history parameter can be fixed by reference to the actual future. Priorian semantics is a formal apparatus, so it is consistent with more than one view about the semantics of natural language. In particular, it is consistent with the claim that, when a future contingent is uttered in a context, the value of the history parameter is fixed in the context as the actual course of events. Instead, if 'PriorianOckhamism' refers to a view about future contingents that may be defended in combination with Priorian semantics, such as contextual pluralism, then it is fair to ask how the history parameter can be fixed. But that question does not essentially concern Priorian semantics ${ }^{27}$.

In general, Priorian semantics must not be confused with contextual pluralism. The distinction between Priorian semantics and contextual puralism is exactly like the distinction between Priorian semantics and Ockhamism: one is a formal apparatus, the other is a view about future contingents. Ockhamism and contextual pluralism are incompatible views about future contingents, but each of them is compatible with Priorian semantics. This suggests that the question of which of the two views is preferable cannot be settled wholly in the arena of formal semantics. Considerations about formal semantics certainly play a crucial role in any accurate discussion of future contingents, but it should not be expected that formal semantics alone can provide a solution to the issue of future contingents. Unfortunately, the formal languages that we can handle are unlike the perfect language imagined by Leibniz, in that they do not allow us to resolve any difference of opinions by means of calculations.

\section{Generalization}

The conclusion that can be drawn from the foregoing sections is that Priorian semantics suits Ockhamism no less than TRL semantics. That is, if A1 and A2 are accepted, no further modification of Priorian semantics is needed. The conditional form of this conclusion implies that what is claimed here

\footnotetext{
${ }^{26}$ Malpass and Wawer [11], p. 122.

${ }^{27}$ Similarly, the objection that Malpass and Wawer raise later in the same section against contextual pluralism, according to which future contingents do not express a complete content, does not essentially concern Priorian semantics.
} 
is not that Priorian semantics is the most appropriate semantics for Ockhamism. Just as Priorian semantics is compatible with more than one view, Ockhamism is compatible with more than one formal semantics. A1 and A2 have not been questioned so far, in that they have been granted as background assumptions. But this does not mean that they are unquestionable. As a matter of fact they are not.

Consider A1. This assumption is often accepted because it is believed that indeterminism - which is taken to be good in itself - entails branching, that is, the conception according to which there is a plurality of possible worlds that overlap up to a certain point, the present. However, it might be argued that, at least on some plausible understanding of indeterminism, indeterminism does not entail branching. Possible futures might be conceived as parts of possible worlds that are wholly distinct, rather than branches that depart from a common trunk ${ }^{28}$.

Consider A2. As the question of how tenses are to be represented hinges on the issue of how logical form is to be individuated, it might be argued that, at least on some plausible understanding of logical form, tenses are not operators. A classical alternative to the representation in terms of operators is the representation in terms of quantification over times. According to the latter, (1) is to be read as 'For some $t$ later than the present time, it rains at $t$ ', (2) is to be read as 'There is a time $t$ such that $t$ is the present time and it rains at $t$ ', and (3) is to be read as 'For some $t$ earlier than the present time, it rains at $t^{\prime 29}$.

Thus, both A1 and A2 might be rejected. It might be contended that an adequate formal semantics for Ockhamism does not need branching time models. Nonetheless, the point made in section 7 could still be acknowledged, that is, Ockhamism can be maintained without assuming that actuality must be formally represented. So the point does not specifically concern Priorian semantics and its relation with TRL semantics ${ }^{30}$.

More generally, this paper suggests that no substantive formal work is needed to equip Ockhamism with an adequate formal semantics. The very idea that a special formal apparatus in which actuality is represented should be tailored to Ockhamism is wrongheaded. This is just one of the many cases in which the search for new formal tools leads to results that are no better than those we could get by using familiar tools we already have ${ }^{31}$.

\footnotetext{
${ }^{28}$ Lewis [9] provides some considerations against branching, pp. 206-209. Iacona [4] includes a discussion of the claim that indeterminism entails branching, pp. 41-44.

${ }^{29}$ Evans [2] is a locus classicus of the early philosophical resistance to tense logic. King [6] is a recent linguistically oriented defence of the representation in terms of quantification over times.

${ }^{30}$ Iacona [5] suggests one way to substantiate the rejection of A1 and A2, in that it outlines a formal apparatus for Ockhamism that is based on the simplest quantified modal logic.

${ }^{31}$ An earlier version of this paper was given as a talk at the University of Leeds in May 2012. I thank those who attended the talk for their insightful questions and comments,
} 


\section{References}

[1] N. Belnap and M. Green. Indeterminism and the Thin Red Line. In J. Tomberlin, editor, Philosophical Perspectives, volume 8, pages 365388. Ridgeview, 1994.

[2] G. Evans. Does Tense Logic Rest upon a Mistake? In Collected Papers, pages 343-363. Clarendon Press, 1985.

[3] Manuel García-Carpintero. Relativism, the Open Future, and Propositional Truth. In F. Correia and A. Iacona, editors, Around the Tree: Semantic and Metaphysical Issues concerning Branching and the Open Future, pages 1-27. Springer, 2012.

[4] A. Iacona. Timeless Truth. In F. Correia and A. Iacona, editors, Around the Tree: Semantic and Metaphysical Issues concerning Branching and the Open Future, pages 29-45. Springer, 2013.

[5] A. Iacona. Ockhamism and Quantified Modal Logic. Logique et Analyse, forthcoming.

[6] J. C. King. Tense, Modality and Semantic Value. Philosophical Perspectives, pages 195-245, 2003.

[7] D. Lewis. Counterfactuals. Blackwell, 1973.

[8] D. Lewis. Anselm and Actuality. In Philosophical Papers, volume I, pages 10-20. Oxford University Press, 1983.

[9] D. Lewis. On the Plurality of Worlds. Blackwell, 1986.

[10] J. MacFarlane. Future Contingents and Relative Truth. Philosophical Quarterly, 53:321-336, 2003.

[11] A. Malpass and J. Wawer. A Future for the Thin Red Line. Synthese, 188:117-142, 2012.

[12] V. R. McKim and C. C. Davis. Temporal Modalities and the Future. Notre Dame Journal of Formal Logic, 17:233-238, 1976.

[13] M. Perloff N. Belnap and M. Xu. Facing the Future. Oxford University Press, 2001.

[14] P. Øhrstrøm. In Defence of the Thin Red Line: a case for Ockhamism. Humana Mente, 8:17-32, 2009.

especially John Divers and Ross Cameron. I'm also grateful to Pablo Rychter and Jordi Valor for the discussions we had on a first draft of the paper, and to two anonymous referees for the helpful revisions they suggested. 
[15] P. Øhrstrøm and P. Hasle. The Stanford Encyclopedia of Philosophy (Summer 2011 Edition), chapter Future Contingents. $\mathrm{URL}=$ ¡tttp://plato.stanford.edu/archives/sum2011/entries/futurecontingents/i, 2011.

[16] A. N. Prior. Past, Present and Future. Clarendon Press, 1967.

[17] S. Rosenkranz. In Defence of Ockhamism. Philosophia, 40:617-31, 2012.

[18] P. T. Braüner, P. Hasle and P. Øhrstrøm. Determinism and the origins of temporal logic. In H. Barringer et al., editor, Advances in Temporal Logic. Kluwer, 2000.

[19] P. Hasle T. Braüner and P. Øhrstrøm. Ockhamistic Logics and True Futures of Counterfactual Moments. In IEEE Press, editor, Proceedings of the Fifth International Workshop on Temporal Representation and Reasoning, pages 132-139, 1998.

[20] R. H. Thomason. Indeterminist Time and Truth-Value Gaps. Theoria, 36:264-281, 1970. 